\title{
Fatores relevantes no processo de permanência prolongada de discentes nos cursos de graduação presencial: um estudo na Universidade Federal do Espírito Santo
}

\author{
Alexandre Severino Pereira a \\ Teresa Cristina Janes Carneiro ${ }^{b}$ \\ Gutemberg Hespanha Brasil c \\ Maria Auxiliadora de Carvalho Corassa ${ }^{d}$
}

\section{Resumo}

A permanência prolongada no ensino superior é a condição em que o estudante demanda um tempo maior do que o previsto na matriz curricular para conclusão do curso. Na Universidade Federal do Espírito Santo, 52,2\% dos estudantes que deveriam se formar nos semestres de 2007/2 a 2012/1 ficaram retidos. O objetivo desta pesquisa foi identificar os fatores associados à retenção nos cursos de graduação presenciais visando propor ações institucionais para reduzir o problema. A metodologia consistiu-se em comparar dois grupos de alunos, os retidos e os formados, por meio de um modelo de regressão logística. Os resultados apontaram 21 fatores intervenientes no processo, dos quais os mais relevantes foram o "coeficiente de rendimento acumulado", o "número de reprovações", a "relação candidato/vaga no vestibular", a "área do conhecimento do curso", o "desempenho na segunda etapa do vestibular" e o "trancamento de curso".

Palavras-chave: Educação superior. Retenção discente. Permanência prolongada. Regressão logística.

\footnotetext{
a Universidade Federal do Espírito Santo - UFES, Divisão de Logística e Infraestrutura da Pró-Reitora de Gestão de Pessoas e Assistência Estudantil. Vitória, Espírito Santo, Brasil.

b Universidade Federal do Espírito Santo - UFES, Mestrado Profissional em Gestão Pública. Coordenadora da Universidade Aberta do Brasil - UAB, Vitória, Espírito Santo, Brasil.

c Universidade Federal do Espírito Santo - UFES, Vitória, Espírito Santo, Brasil.

d Universidade Federal do Espírito Santo - UFES, Pró-reitora de Graduação. Vitória, Espírito Santo, Brasil. 


\section{Introdução}

Para realização deste estudo, a retenção ou permanência prolongada foi definida como a condição em que o estudante demanda um tempo maior do que o previsto na matriz curricular para integralização da carga horária do curso.

Essa permanência prolongada significa um tempo maior do que o planejado pela instituição para a conclusão do curso, o que compromete a taxa de sucesso, gera ociosidade de recursos humanos e materiais e pode provocar a evasão do estudante. Dessa forma, a retenção deve ser vista como grave problema no processo de ensino, em diversas perspectivas: do estudante, pois causa prejuízos de ordem pessoal, profissional e financeira; da instituição, por comprometer a eficiência e produtividade do sistema, comprometer a taxa de conclusão e de ocupação das vagas e gerar custos adicionais à universidade; e da sociedade, retardando a disponibilização de cidadãos capacitados para o mercado de trabalho e reduzindo o retorno social ligado à formação de profissionais de nível superior.

Um estudo realizado em 2013 na Universidade Federal do Espírito Santo (UFES) apontou que $52,2 \%$ dos estudantes que deveriam se formar nos semestres de 2007/2 a 2012/1 ficaram retidos (PEREIRA, 2013).

Em função da importância dos efeitos decorrentes dos fenômenos de evasão e retenção, foi constatada a necessidade de realização de uma pesquisa para conhecimento dos fatores associados a esses processos no âmbito das instituições federais de ensino superior brasileiras, e esta pesquisa é parte dessa iniciativa. Assim, o objetivo deste estudo foi identificar os fatores associados à permanência prolongada dos estudantes dos cursos de graduação presenciais de uma Universidade Federal.

Para isso, a primeira etapa do trabalho consistiu-se em buscar teorias para fundamentação do estudo. Dessa busca foi possível observar que a maior parte dos modelos teóricos existentes trata da permanência ou da evasão, sem referências à retenção (prolongamento do curso). No entanto, é razoável considerar que os processos de permanência prolongada e evasão apresentam estreita relação entre si, tendo em vista que ambos ocorrem no ambiente universitário e envolvem os mesmos tipos de atores (estudantes, professores, familiares, colegas e outros envolvidos). Dessa forma, entende-se que os referidos modelos podem ser aplicados a estudos de permanência prolongada, desde que observadas as características e restrições de cada teoria. 
Dentre os modelos teóricos existentes, o Modelo Conceitual de Desgaste de Estudantes Não Tradicionais, proposto por Bean e Metzner (1985), foi utilizado para fundamentar a definição do modelo a ser adotado para condução deste estudo. Embora a referida teoria tenha sido adotada como referência para orientação da pesquisa, outros modelos teóricos (ASTIN, 1984; BEAN, 1980; CABRERA et al., 1992; PASCARELLA, 1980; SPADY, 1970, 1971; TINTO, 1975, 1993, 1997) podem fornecer contribuições para a compreensão do fenômeno.

A principal preocupação de Bean e Metzner (1985) em seu modelo conceitual foi criar uma estrutura de relações que fosse capaz de explicar o processo de desgaste que leva o estudante não tradicional a abandonar o curso (evadir). Os autores consideraram como estudantes não tradicionais aqueles que não se restringem ao estereótipo dos estudantes tradicionais norte-americanos (jovens, brancos, filhos de pais com formação superior e com dedicação exclusiva aos estudos em instituições residenciais).

Bean e Metzner (1985) descrevem o modelo indicando que a decisão de evadir é baseada essencialmente em quatros fatores. O primeiro e mais importante é o desempenho acadêmico que, por sua vez, é influenciado fortemente pelos resultados acadêmicos passados. O segundo fator é a intenção de sair, a qual é influenciada pelos resultados psicológicos (utilidade, satisfação, compromisso com o objetivo) e pelas variáveis acadêmicas (hábitos de estudos, aconselhamento acadêmico, convicção de formação, absenteísmo). O terceiro grupo de variáveis é a experiência e variáveis de definição (idade, etnia, gênero), principalmente o desempenho no ensino médio e objetivos educacionais. O quarto fator são as variáveis ambientais (situação financeira, horas de trabalho, responsabilidades familiares, oportunidade de transferência, encorajamento de familiares e amigos), das quais se espera substancial efeito direto sobre a decisão de evadir. Além dessas variáveis, os autores incluíram possíveis influências de integração social e de efeitos mediadores compensatórios entre as variáveis acadêmicas e ambientais e entre os resultados acadêmicos e psicológicos no processo.

Os autores destacaram ainda as variáveis ambientais (representadas pela carga de trabalho profissional, responsabilidades familiares e questões de ordem financeira) e sua influência como importante contribuição para compreensão do processo de evasão de estudantes não tradicionais.

Além dos modelos teóricos revisados, alguns estudos realizados no Brasil contribuíram para avanço do conhecimento sobre o processo de permanência prolongada. Campello e Lins (2008), Lautert, Rolim e Loder (2011) e Noronha, Carvalho e Santos (2001), 
apontaram que a realização de estágios é uma das principais causas da retenção discente. Já Campello e Lins (2008) e Polydoro (2000) relataram que os trancamentos de cursos causaram o prolongamento da permanência do aluno.

Rios, Santos e Nascimento (2001) indicaram que um baixo desempenho nas provas discursivas do vestibular tem relação com baixo desempenho no início do curso e, consequentemente, pode levar o aluno à retenção. Corrêa e Noronha (2004) e Campello e Lins (2008) apontaram que as reprovações e realização de intercâmbio são importantes causadores da permanência prolongada.

\section{Metodologia}

Esta pesquisa adotou abordagem quantitativa com características de estudo descritivo (quanto aos objetivos), tendo em vista que buscou descobrir se existem e em que nível existem associações entre diversas variáveis e o processo de permanência prolongada. Os dados utilizados foram obtidos de bancos de dados da instituição (dados secundários).

O local de realização do estudo foi a Universidade Federal do Espírito Santo (UFES), que em 2013 possuía cerca de 20 mil estudantes matriculados em 94 cursos de graduação oferecidos em quatro campi universitários. Mais informações sobre a instituição podem ser obtidas no portal eletrônico http://portal.ufes.br/.

O foco deste estudo foi a identificação de fatores associados ao fenômeno da permanência prolongada de discentes nos cursos de graduação oferecidos na modalidade presencial, não foram avaliados os cursos de pós-graduação nem os cursos de graduação na modalidade a distância.

\subsection{Modelo teórico proposto para o estudo}

Para proposição do modelo aplicado neste estudo foi utilizado o Modelo Conceitual de Desgaste de Estudantes Não Tradicionais de Bean e Metzner (1985). O referido modelo foi considerado mais adequado ao caso estudado, tendo em vista que não se restringe ao estereótipo do estudante tradicional norte-americano. A Figura apresenta o modelo proposto para o estudo.

Nesse modelo proposto considerou-se que as "Características pessoais e fatores anteriores ao ingresso", o "Contexto familiar e condições financeiras", o "Ambiente Institucional" e "Desempenho acadêmico" podem afetar diretamente a permanência prolongada. Possíveis relações entre os grupos de variáveis explicativas como indicado no modelo original de Bean e Metzner (1985) não foram analisadas neste estudo. 


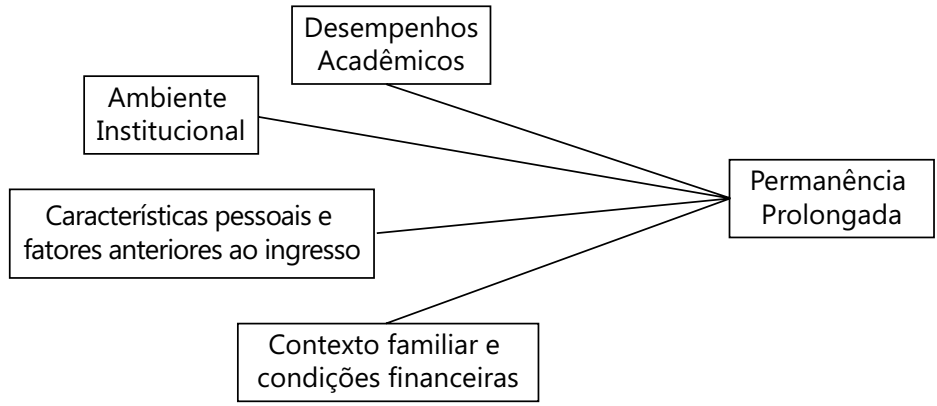

Fonte: Elaborada pelos autores (2013).

Figura. Modelo proposto para estudo dos fatores associados ao processo de retenção.

Em função das características do local de estudo e questões ligadas à obtenção dos dados, três variáveis do modelo original foram desconsideradas (carga horária, habilidade de estudo e oportunidades de transferência) e outra foi substituída (desempenho no segundo grau por desempenho no vestibular). Além disso, novas variáveis foram incluídas, tendo em vista o interesse de explorar possíveis associações.

A nomenclatura dos grupos de variáveis adotada neste estudo é diferente daquela usada no modelo original, pois se buscou utilizar nomes que expressassem adequadamente aquilo que representam. Desse modo, "Características pessoais e fatores anteriores ao ingresso" equivale a "Experiência e variáveis de definição", "Contexto familiar e condições financeiras" substituiu "Variáveis Ambientais", "Ambiente Institucional" corresponde a "Variáveis Acadêmicas" e "Desempenho Acadêmico" representa "Resultados Acadêmicos".

\subsection{Obtenção dos dados}

Parte dos dados utilizados na pesquisa foi solicitada a setores da instituição (Comissão Coordenadora do Vestibular, Pró-reitorias de Graduação, Extensão, Pesquisa e Pós-Graduação, Gestão de Pessoas e Assistência Estudantil e Núcleo de Processamento de Dados) e a outra foi extraída diretamente de relatórios disponíveis no Sistema de Informações para o Ensino (SIE).

As fontes de dados utilizadas foram o cadastro do SIE, o cadastro e Questionário Socioeconômico do Vestibular, o cadastro dos Programas de Iniciação Científica e Assistência Estudantil e o Sistema de Estágio. O detalhamento das variáveis constantes no modelo proposto é apresentado no Quadro. 
Quadro. Detalhamento das variáveis do modelo proposto para estudo dos fatores associados ao processo de retenção.

\begin{tabular}{|c|c|c|}
\hline Grupo de Variáveis & Variável Conceitual & Variável Operacional \\
\hline Dependente / Resposta & Situação final do aluno & $\begin{array}{l}\text { Situação final do aluno (retido ou } \\
\text { formado) }\end{array}$ \\
\hline \multirow{16}{*}{$\begin{array}{l}\text { Características } \\
\text { pessoas e fatores } \\
\text { anteriores ao ingresso }\end{array}$} & \multirow[t]{3}{*}{ Características pessoais } & Sexo / Gênero \\
\hline & & Faixa etária \\
\hline & & Cor / etnia \\
\hline & \multirow[t]{3}{*}{ Escolaridade anterior } & Escola do ensino fundamental \\
\hline & & Escola do ensino médio \\
\hline & & Situação em relação a curso superior \\
\hline & Motivo para escolha do curso & Motivo para escolha do curso \\
\hline & Objetivos educacionais & O que espera do curso \\
\hline & \multirow[t]{8}{*}{ Vestibular } & Relação candidato/vaga do vestibular \\
\hline & & Desempenho no vestibular ( $2^{\mathrm{a}}$ etapa) \\
\hline & & Opção por Cotas \\
\hline & & Isenção de taxa do vestibular \\
\hline & & Curso preparatório para o vestibular \\
\hline & & Participação em processos seletivos \\
\hline & & $\begin{array}{l}\text { Meio de comunicação para manter-se } \\
\text { informado }\end{array}$ \\
\hline & & Tentativas de ingresso na UFES \\
\hline \multirow{12}{*}{$\begin{array}{l}\text { Ambiente } \\
\text { Institucional }\end{array}$} & Área do Conhecimento & Área do Conhecimento \\
\hline & \multirow{2}{*}{$\begin{array}{l}\text { Envolvimento com Iniciação } \\
\text { Científica/Pesquisa }\end{array}$} & Participação no PIBIC \\
\hline & & Participação no PIVIC \\
\hline & Ajuda financeira & Assistência Estudantil \\
\hline & Realização de estágio & Meses de estágio \\
\hline & Aproveitamento de disciplinas & $\begin{array}{l}\text { Quantidade de disciplinas } \\
\text { aproveitadas }\end{array}$ \\
\hline & Realização de intercâmbio & Realização de intercâmbio cultural \\
\hline & Trancamento de curso & Semestres de trancamento de curso \\
\hline & \multirow[t]{4}{*}{ Curso } & Centro Acadêmico \\
\hline & & Tempo esperado para conclusão \\
\hline & & Ano de início do curso \\
\hline & & Turno do curso \\
\hline
\end{tabular}

Continua 


\begin{tabular}{|c|c|c|}
\hline \multicolumn{3}{|l|}{ Continuação } \\
\hline \multirow{15}{*}{\begin{tabular}{l|} 
Contexto Familiar e \\
Condições Financeiras
\end{tabular}} & \multirow[t]{4}{*}{ Condições financeiras } & Renda mensal da família \\
\hline & & $\begin{array}{c}\text { Número de pessoas do grupo } \\
\text { familiar }\end{array}$ \\
\hline & & Número de carros de passeio \\
\hline & & Tipo de atividade remunerada \\
\hline & \multirow[t]{2}{*}{ Responsabilidade Familiar } & Estado Civil \\
\hline & & $\begin{array}{l}\text { Participação na vida econômica da } \\
\text { família }\end{array}$ \\
\hline & \multirow[t]{9}{*}{ Contexto Familiar } & Região (Município) de origem \\
\hline & & Nível de instrução do pai \\
\hline & & Nível de instrução da mãe \\
\hline & & Ocupação do pai ou responsável \\
\hline & & Ocupação da mãe \\
\hline & & Situação empregatícia do pai \\
\hline & & Situação empregatícia da mãe \\
\hline & & Tipo de moradia do candidato/aluno \\
\hline & & Tipo de moradia dos pais \\
\hline \multirow[t]{2}{*}{$\begin{array}{l}\text { Desempenho } \\
\text { Acadêmico }\end{array}$} & \multirow[t]{2}{*}{ Resultados formais } & $\begin{array}{l}\text { Coeficiente de rendimento } \\
\text { acumulado }\end{array}$ \\
\hline & & Número de reprovações \\
\hline
\end{tabular}

Nota: PIBIC - Programa Institucional de Bolsas de Iniciação Científica; PIVIC - Programa Institucional de Voluntários de Iniciação Científica.

Fonte: Elaborado pelos autores (2013).

\subsection{Amostra}

A amostra de estudo foi obtida com a aplicação da metodologia utilizada pela Comissão Especial de Estudos sobre a Evasão (BRASIL, 1997), denominada Fluxo (ou Acompanhamento de Estudantes). A metodologia foi aplicada a quatro gerações completas, cujos prazos esperados de integralização curricular terminavam nos semestres 2010/2, 2011/1,2011/2 e 2012/1. Cursos que começaram a funcionar num período inferior ao prazo esperado de integralização não foram incluídos, por não possuírem gerações completas.

Com base nessa metodologia, os seguintes procedimentos foram adotados: (1) Obtenção dos dados dos alunos ingressantes e evadidos (inclusive diplomados); (2) Recuperação do número de períodos sugeridos por curso e versão do currículo';

1 "Versão do currículo" refere-se à versão da matriz curricular do curso vigente quando do ingresso do aluno. 
(3) Identificação dos alunos cujo prazo esperado de conclusão terminou nos semestres 2010/2, 2011/1, 2011/2 e 2012/1 (gerações completas); (4) Verificação da situação do aluno no semestre limite de conclusão do curso, segundo o seu ano/período de ingresso e a duração sugerida na matriz curricular.

Na sequência foi verificada a disponibilidade de dados do vestibular e do histórico escolar dos selecionados. Para os casos de indisponibilidade dos referidos dados, o aluno foi excluído da amostra. Por fim, os alunos evadidos (não diplomados) até o ano/período esperado de conclusão também foram excluídos.

Desse modo, a amostra obtida continha 4.947 alunos, sendo 1.163 (23,5\%) da primeira geração (conclusão esperada em 2010/2), 1.218 (24,6\%) da segunda (conclusão esperada em 2011/1), 1.273 (25,7\%) da terceira (conclusão esperada em 2011/2) e 1.293 (26,2\%) da quarta (conclusão esperada em 2012/1). Em relação à situação final, $1.831(37,0 \%)$ alunos formaram-se no tempo esperado e $3.116(63,0 \%)$ ficaram retidos.

A maioria dos alunos da amostra é do sexo feminino (51,6\%). A média de idade do grupo (na época da realização do vestibular) era de 20 anos, variando de 16 a 57 anos, dos quais $2.304(46,6 \%)$ alunos tinham 18 anos ou menos, $1.552(31,4 \%)$ tinham entre 19 e 20 anos e 1.091 (22,0\%) tinham 21 anos ou mais. Quanto ao estado civil, à época do vestibular, $93,7 \%$ eram solteiros e $4,0 \%$ casados.

\subsection{Modelo de regressão logística}

A Regressão Logística é uma técnica aplicada para estimar a probabilidade de um evento ocorrer e para identificar características de indivíduos ou elementos que pertencem a cada grupo definido com base em uma variável categórica (FÁVERO et al., 2009).

Embora a técnica permita a realização de previsões, neste estudo buscou-se identificar as variáveis independentes que conjuntamente influenciam a chance de um aluno ficar retido, revelando aquelas que são relevantes (significantes) no processo.

O passo inicial da aplicação da técnica consistiu na definição das variáveis que foram utilizadas. A "Situação final do aluno" (retido ou formado) foi utilizada como variável dependente ou resposta, enquanto as variáveis independentes ou explicativas foram as 46 variáveis categóricas pertencentes aos quatro grupos incluídos no modelo teórico proposto para o estudo (seção 2.1).

Em seguida foram definidas as categorias de referência das variáveis explicativas, que serviram de base para a análise, utilizando os resultados de Testes de 
Independência de Qui-quadrado de Pearson. Para cada variável independente foi definida, como categoria de referência, aquela que possuía maior chance de ser característica de alunos não retidos, com exceção das variáveis que indicavam presença ou ausência de um atributo (sim ou não), nas quais definiu-se como referência as categorias relativas à ausência (não).

Optou-se pela utilização do método Backward stepwise do software SPSS 2 (versão 20) e da estatística teste Razão de Verossimilhança. O nível de significância adotado foi de $5,0 \%$, inclusive como critério para entrada ou saída de variáveis no processo stepwise. O ponto de corte para a classificação dos alunos foi igual a $0,5(50 \%)$.

Como etapa preliminar à análise da técnica de Regressão Logística, foi verificada a existência de alta correlação entre variáveis explicativas, a fim de evitar problemas de multicolinearidade, considerando como critério os valores do coeficiente de correlação linear de Pearson (r) maiores que 0,7 ou menores que -0,7 (FÁVERO et al., 2009).

A avaliação da qualidade do ajuste do modelo e precisão preditiva foi baseada nas medidas indicadas por Corrar, Paulo e Dias Filho (2007), Fávero et al. (2009) e Hair et al. (2009).

Após a verificação da qualidade do ajustamento, foi analisado o modelo logístico gerado pela técnica, onde constam as variáveis significantes e relevantes no processo de retenção dos estudantes dos cursos de graduação presencial da universidade.

Foram analisados os graus de significância dos coeficientes das variáveis do modelo logístico, utilizando-se a estatística Wald (CORRAR; PAULO; DIAS FILHO, 2007). Para os coeficientes logísticos significantes, foi possível avaliar o quanto impactam sobre a probabilidade estimada e a previsão de pertinência a um grupo (ou situação) com o uso do coeficiente logístico exponenciado, comumente conhecido como exp(B) ou odds ratio (HAIR et al., 2009).

Esses autores indicam que um coeficiente exponenciado maior que 1,0 representa uma relação positiva entre a variável explicativa e a variável resposta, enquanto valores menores que 1,0 correspondem a uma relação negativa. Já em relação à magnitude, a variação da probabilidade (razão de desigualdade) de ocorrência do evento pode ser verificada com base na diferença entre o valor do coeficiente exponenciado e a unidade (HAIR et al., 2009). A interpretação dos coeficientes exponenciados deve considerar seu efeito multiplicativo sobre a razão de chances (ou de risco).

\footnotetext{
2 Statistical Package for the Social Sciences (Field, 2009).
} 


\section{Resultados e discussão}

\subsection{Fatores relevantes no processo de retenção}

Inicialmente, para evitar a ocorrência de multicolinearidade foram avaliadas as correlações entre as variáveis explicativas. No primeiro modelo logístico gerado pela técnica foi observada a existência de alta correlação entre a variável "Tempo esperado para conclusão" e o termo Constante $(\mathrm{r}=-0,947)$ e entre as variáveis "Centro Acadêmico" e "Área do Conhecimento" $(\mathrm{r}=-0,942)$. Diante desse fato, optou-se pela permanência do termo Constante, com base no princípio da parcimônia, e da variável "Área do Conhecimento", por não ser restrita à instituição pesquisada, e consequentemente, exclusão das outras duas.

Na sequência, um novo modelo logístico foi gerado e a análise dos coeficientes indicou ausência de alta correlação entre variáveis remanescentes, e então se avançou para a análise do modelo. O processamento do modelo foi baseado em $4.616(93,3 \%)$ indivíduos da amostra, tendo em vista que em $331(6,7 \%)$ casos observou-se ausência de algum dado.

O modelo final foi considerado significante (Teste de Significância dos Coeficientes do Modelo - p-valor $=0,000$ ). Aproximadamente 64,8\% das variações registradas na variável dependente podem ser explicadas pelo modelo ( $\mathrm{R}^{2}$ de Nagelkerke). Além disso, não existem diferenças significativas entre os resultados preditos e os observados (Teste de Hosmer e Lemeshow - p-valor $=0,603)$ e o modelo gerado possui excelente poder discriminatório (área sob a Curva $\mathrm{ROC}^{3}=0,923$ ). Em termos de acurácia, o modelo foi capaz de classificar corretamente $80,0 \%$ dos formados e $88,0 \%$ dos retidos, resultando em uma precisão geral de $85,0 \%$.

Das 44 variáveis explicativas utilizadas (todas categóricas), 21 foram consideradas significantes, ao nível de 5,0\%, para compor o modelo logístico final produzido pela técnica e podem ser considerados os fatores mais relevantes associados com a retenção dos alunos nos cursos de graduação da universidade. Esses fatores são também aqueles que melhor discriminam os alunos retidos em relação aos formados (diplomados).

Desse conjunto de variáveis significantes: seis são relativas a "Características pessoais e fatores anteriores ao ingresso", quatro são do grupo "Contexto

3 Receiver Operating Characteristic. 
familiar e condições financeiras" (fatores externos a instituição), nove são do "Ambiente Institucional" (fatores internos à instituição) e duas representam "Desempenho Acadêmico".

A Tabela apresenta as variáveis significantes mantidas no modelo logístico, suas categorias, nível de significância (p-valor), coeficientes logísticos exponenciados $(\operatorname{Exp}(B))$ e intervalo de confiança de $95 \%$ (I.C.) para os valores de $\operatorname{Exp}(\mathrm{B})$.

Tabela. Regressão Logística - Método Backward Stepwise - Variáveis mantidas na equação.

\begin{tabular}{|c|c|c|c|c|c|}
\hline \multirow{2}{*}{ Variável (Grupo) } & \multirow{2}{*}{ Categoria } & \multirow{2}{*}{ p-valor } & \multirow{2}{*}{$\operatorname{Exp}(B)$} & \multicolumn{2}{|c|}{ I.C. para EXP(B) } \\
\hline & & & & Inferior & Superior \\
\hline \multirow{2}{*}{$\begin{array}{l}\text { Opção no sistema } \\
\text { de cotas }(C P)\end{array}$} & Não optante (Ref.) & & & & \\
\hline & Optante & 0,000 & 4,605 & 2,670 & 7,942 \\
\hline \multirow{5}{*}{$\begin{array}{l}\text { Relação } \\
\text { candidatos/vaga } \\
\text { (CP) }\end{array}$} & Maior que 9,44 (Ref.) & 0,000 & & & \\
\hline & Até 3,30 & 0,000 & 4,118 & 2,824 & 6,005 \\
\hline & Entre 3,31 e 4,85 & 0,000 & 2,059 & 1,462 & 2,899 \\
\hline & Entre 4,86 e 7,13 & 0,000 & 1,800 & 1,339 & 2,418 \\
\hline & Entre 7,14 e 9,44 & 0,000 & 2,101 & 1,554 & 2,841 \\
\hline \multirow{3}{*}{$\begin{array}{l}\text { O que espera em } \\
1^{\circ} \text { lugar de um } \\
\text { curso superior }(C P)\end{array}$} & Qualificação para profissão (Ref.) & 0,018 & & & \\
\hline & Compreender melhor o mundo & 0,013 & 1,498 & 1,088 & 2,063 \\
\hline & Melhorar o nível de instrução & 0,009 & 1,422 & 1,093 & 1,851 \\
\hline \multirow{2}{*}{$\begin{array}{l}\text { Motivo } \\
\text { predominante na } \\
\text { escolha do curso } \\
\text { (CP) }\end{array}$} & Possibilidades de emprego (Ref.) & 0,018 & & & \\
\hline & $\begin{array}{l}\text { Curso adequado à sua aptidão } \\
\text { pessoal e vocacional }\end{array}$ & 0,012 & 1,484 & 1,091 & 2,017 \\
\hline \multirow{3}{*}{$\begin{array}{l}\text { Desempenho no } \\
\text { Vestibular (CP) }\end{array}$} & Mais de 6,00 pontos (Ref.) & 0,000 & & & \\
\hline & Até 3,00 pontos & 0,001 & 2,077 & 1,371 & 3,145 \\
\hline & Entre 3,01 e 6,00 pontos & 0,000 & 2,088 & 1,511 & 2,884 \\
\hline \multirow{2}{*}{$\begin{array}{l}\text { Situação em } \\
\text { relação a um curso } \\
\text { superior (CP) }\end{array}$} & $\begin{array}{l}\text { Nunca iniciou um curso de nível } \\
\text { superior (Ref.) }\end{array}$ & 0,048 & & & \\
\hline & Já o concluiu & 0,014 & 2,625 & 1,217 & 5,664 \\
\hline \multirow{3}{*}{$\begin{array}{l}\text { Região de origem } \\
\text { (CF) }\end{array}$} & Grande Vitória (Ref.) & 0,001 & & & \\
\hline & Interior ES & 0,010 & 0,718 & 0,558 & 0,924 \\
\hline & Outros Estados & 0,001 & 0,465 & 0,292 & 0,738 \\
\hline \multirow{2}{*}{$\begin{array}{l}\text { Ocupação do pai } \\
\text { ou responsável (CF) }\end{array}$} & Agricultor/Empregado rural (Ref.) & 0,014 & & & \\
\hline & Servidor público & 0,043 & 1,553 & 1,014 & 2,377 \\
\hline
\end{tabular}

Continua 
Continuação

\begin{tabular}{|c|c|c|c|c|c|}
\hline \multirow{3}{*}{$\begin{array}{l}\text { Situação } \\
\text { empregatícia do } \\
\text { pai (CF) }\end{array}$} & Trabalha (Ref.) & 0,002 & & & \\
\hline & Está desempregado & 0,021 & 1,600 & 1,073 & 2,386 \\
\hline & $\begin{array}{c}\text { É aposentado e não está } \\
\text { trabalhando }\end{array}$ & 0,000 & 1,951 & 1,377 & 2,763 \\
\hline \multirow{3}{*}{$\begin{array}{l}\text { Número de } \\
\text { pessoas que } \\
\text { vivem dessa renda } \\
\text { familiar (CF) }\end{array}$} & Uma (Ref.) & 0,026 & & & \\
\hline & Duas & 0,017 & 0,397 & 0,186 & 0,846 \\
\hline & Quatro & 0,021 & 0,440 & 0,219 & 0,884 \\
\hline \multirow[t]{2}{*}{ Trancamentos (AI) } & Nenhum trancamento (Ref.) & 0,000 & & & \\
\hline & Um semestre & 0,000 & 15,103 & 7,642 & 29,848 \\
\hline \multirow{3}{*}{$\begin{array}{l}\text { Número de } \\
\text { disciplinas } \\
\text { aproveitadas (AI) }\end{array}$} & Cinco disciplinas ou mais (Ref.) & 0,000 & & & \\
\hline & Nenhuma disciplina & 0,000 & 2,452 & 1,861 & 3,232 \\
\hline & De duas a quatro disciplinas & 0,001 & 1,726 & 1,263 & 2,360 \\
\hline \multirow{2}{*}{$\begin{array}{l}\text { Participação no } \\
\text { PIBIC (AI) }\end{array}$} & Não (Ref.) & & & & \\
\hline & Sim & 0,020 & 0,699 & 0,517 & 0,945 \\
\hline \multirow{2}{*}{$\begin{array}{l}\text { Participação no } \\
\text { PIVIC (AI) }\end{array}$} & Não (Ref.) & & & & \\
\hline & Sim & 0,001 & 2,146 & 1,369 & 3,365 \\
\hline \multirow{2}{*}{$\begin{array}{l}\text { Realização de } \\
\text { estágio (AI) }\end{array}$} & Sem estágio (Ref.) & 0,035 & & & \\
\hline & De 12 a 24 meses & 0,012 & 0,714 & 0,549 & 0,929 \\
\hline \multirow{6}{*}{$\begin{array}{l}\text { Área do } \\
\text { conhecimento (AI) }\end{array}$} & Ciências da Saúde (Ref.) & 0,000 & & & \\
\hline & Ciências Agrárias & 0,000 & 0,404 & 0,251 & 0,652 \\
\hline & Ciências Biológicas & 0,000 & 3,182 & 1,998 & 5,065 \\
\hline & Ciências Humanas & 0,000 & 2,148 & 1,484 & 3,110 \\
\hline & Ciências Sociais Aplicadas & 0,001 & 1,736 & 1,257 & 2,397 \\
\hline & Linguística, Letras e Artes & 0,000 & 7,196 & 4,263 & 12,145 \\
\hline \multirow{2}{*}{$\begin{array}{l}\text { Ano de início de } \\
\text { funcionamento do } \\
\text { curso (AI) }\end{array}$} & A partir de 2006 (Ref.) & 0,000 & & & \\
\hline & De 1990 a 2005 & 0,000 & 3,608 & 2,445 & 5,324 \\
\hline \multirow{2}{*}{$\begin{array}{l}\text { Intercâmbio } \\
\text { cultural (AI) }\end{array}$} & Não (Ref.) & & & & \\
\hline & Sim & 0,000 & 11,278 & 3,957 & 32,143 \\
\hline \multirow{2}{*}{$\begin{array}{l}\text { Turno do curso } \\
\text { (AI) }\end{array}$} & Diurno (Ref.) & & & & \\
\hline & Noturno & 0,005 & 1,597 & 1,153 & 2,212 \\
\hline \multirow{5}{*}{$\begin{array}{l}\text { Coeficiente de } \\
\text { rendimento } \\
\text { acumulado (DA) }\end{array}$} & CRA de 9,01 a 10,00 (Ref.) & 0,000 & & & \\
\hline & CRA de 4,01 a 6,00 & 0,000 & 11,152 & 6,177 & 20,135 \\
\hline & CRA de 6,01 a 7,00 & 0,000 & 6,142 & 4,101 & 9,200 \\
\hline & CRA de 7,01 a 8,00 & 0,000 & 4,866 & 3,608 & 6,561 \\
\hline & CRA de 8,01 a 9,00 & 0,000 & 1,970 & 1,581 & 2,455 \\
\hline \multirow{4}{*}{$\begin{array}{l}\text { Número de } \\
\text { reprovações (DA) }\end{array}$} & Nenhuma reprovação (Ref.) & 0,000 & & & \\
\hline & Uma reprovação & 0,000 & 2,293 & 1,794 & 2,930 \\
\hline & De duas a cinco reprovações & 0,000 & 4,876 & 3,777 & 6,294 \\
\hline & Seis reprovações ou mais & 0,000 & 41,704 & 26,052 & 66,758 \\
\hline \multicolumn{2}{|l|}{ Constante } & 0,000 & 0,017 & & \\
\hline
\end{tabular}

CP: Características pessoais e fatores anteriores ao ingresso; CF: Contexto familiar e condições financeiras; AI: Ambiente Institucional; DA: Desempenho Acadêmico; I.C.: Intervalo de confiança de 95\%; Ref.: Categoria de referência; CRA: Coeficiente de rendimento acumulado.

Nota: Foram mantidas somente as categorias significantes ao nível de $5 \%$ ( $p$-valor $<0,050$ ). Fonte: Elaborada pelos autores (2013). 
A partir dos dados contidos na Tabela são apresentadas as variações nas chances de retenção de alunos em função de mudanças nas variáveis explicativas. Para tanto, ressalta-se que as interpretações são realizadas considerando que as demais variáveis explicativas são controladas, ou seja, não sofrem variações.

\subsubsection{Características pessoais e fatores anteriores ao ingresso (CP)}

O aluno optante pelo sistema de cotas ("cotista") tem maior risco de retenção - a chance desse aluno ficar retido é 4,6 vezes a do aluno não optante. Um importante dado refere-se ao intervalo de confiança do valor do coeficiente exponenciado. No caso do aluno optante estima-se que em $95 \%$ dos casos o valor real da chance de retenção de um membro desse grupo é pelo menos 2,6 vezes a do não optante, podendo chegar a ser 7,9 vezes.

Já a "relação candidato/vaga" no vestibular de ingresso possui relação negativa com a chance de retenção, tendo em vista que, em geral, menores valores da variável representam maior risco de retenção. Os resultados obtidos indicam que o aluno que prestou vestibular para um curso com concorrência de até 3,3 candidatos por vaga no vestibular possui 4,1 vezes o risco daquele que a relação candidato por vaga era superior a 9,44. Nas faixas intermediárias de valores da variável explicativa, o risco de retenção também foi maior do que da faixa de maior concorrência.

Os estudantes que indicaram que pretendiam "compreender melhor o mundo" ou "melhorar o nível de instrução" como principal expectativa em relação ao curso superior possuem, respectivamente, 1,5 e 1,4 vezes a chance de retenção dos indivíduos que esperavam "qualificação profissional".

Na pergunta (variável) "Motivo predominante na escolha do curso", observou-se que os discentes que indicaram a opção "Curso adequado a sua aptidão pessoal e vocacional" possuem aproximadamente 1,5 vezes o risco de ficarem retidos daqueles que indicaram a opção "Possibilidade de emprego".

Sobre a variável "Situação em relação a um curso superior", o modelo logístico aponta que o aluno que respondeu que "já concluiu" uma graduação possui 2,6 vezes a chance de retenção daquele que "nunca iniciou um curso superior".

Os alunos que obtiveram um "desempenho no vestibular" (média aritmética das notas das provas da $2^{\mathrm{a}}$ etapa) de até 6,00 pontos possuem o dobro do risco de ficarem retidos do que os que alcançaram um desempenho entre 6,01 e 10,00 pontos. 


\subsubsection{Contexto familiar e condições financeiras (CF)}

Em relação à "Região de origem", o modelo logístico indica que os alunos originários do interior do Espírito Santo e de outros estados têm, respectivamente, $28,2 \%$ e $53,5 \%$ menores chances de ficarem retidos que aqueles dos municípios da Grande Vitória.

O aluno cujo pai ou responsável é "servidor público" possui 1,5 vezes a chance de retenção do que outro que seja dependente de um "agricultor ou trabalhador rural".

O risco de um aluno cujo pai é "aposentado ou não está trabalhando" (na época do vestibular de ingresso) é 95,1\% maior do que de outro cujo pai "trabalha". E quando o pai está desempregado essa chance é $60 \%$ maior.

O estudante que compõe um grupo familiar (variável "número de pessoas que vivem da renda familiar") de "duas" ou de "quatro pessoas" possui, respectivamente, $60,3 \%$ e $56,0 \%$ menos chances de ficar retido do que aquele de vive sozinho.

\subsubsection{Ambiente institucional (AI)}

A chance de ficar retido de um aluno que trancou o curso por um semestre é 15,1 vezes a do discente com nenhum trancamento. Neste caso, com um nível de confiança de $95 \%$, essa chance de retenção varia de 7,6 a 29,8 vezes, ou seja, a chance de um discente que trancou o curso é no mínimo 7,6 vezes a daquele que não trancou.

Os estudantes que não aproveitaram disciplinas possuem chance de retenção de 2,4 vezes a dos que aproveitaram "cinco disciplinas ou mais". Para os alunos que aproveitaram de "duas a quatro disciplinas", essas chances são 1,7 vezes.

A associação da participação do aluno nos programas de iniciação científica e a permanência prolongada foi investigada através duas variáveis. Uma delas, "participação no PIBIC" (Programa Institucional de Bolsas de Iniciação Científica), apresenta associação negativa com a retenção, de forma que o aluno que "participou" do programa possui 30,1\% menos chances de ficar retido do que aquele que "não participou”. Já a "participação no PIVIC" (Programa Institucional de Voluntários de Iniciação Científica) demonstrou-se positivamente associada com a retenção e o risco do discente "participante" deste programa é 2,1 vezes a de um "não participante". 
Os alunos que realizaram estágio por períodos entre 12 e 24 meses possuem $28,6 \%$ menor risco de retenção do que aqueles que não estagiaram.

Os alunos dos cursos da área de "Linguística, Letras e Artes" possuem chance de retenção de 7,2 vezes a dos alunos da área de referência ("Ciências da Saúde"), assim como os alunos dos cursos das áreas de "Ciências Biológicas" (3,1 vezes), "Ciências Humanas" (2,1 vezes) e "Ciências Sociais Aplicadas" (1,7 vezes) também possuem maior risco de retenção. Já a área de "Ciências Agrárias" possui 59,6\% menos chance de retenção do que a categoria de referência. As áreas de "Ciências Exatas e da Terra e Engenharias" não atingiram o nível de significância adotado.

A associação do tempo de funcionamento do curso com o risco de retenção do aluno foi avaliada com base na variável "ano de início de funcionamento do curso". Três categorias foram utilizadas, uma para representar os cursos mais recentes ("a partir de 2006"), uma para os cursos mais antigos ("antes de 1990") e uma para os cursos em fase intermediária de tempo de funcionamento ("de 1990 a 2005"). Para esta variável, o modelo logístico indicou que os alunos dos cursos iniciados entre " 1990 e 2005 " possuem 3,6 vezes o risco de ficarem retidos daqueles que frequentam cursos mais recentes (início de funcionamento a partir de 2006).

O risco de ficar retido para o aluno que realizou intercâmbio cultural é 11,2 vezes a do que não realizou, sendo (com nível de confiança de 95\%) pelo menos 3,9 vezes e podendo chegar a ser 32,1 vezes. Quanto ao turno do curso, os alunos dos cursos noturnos possuem 59,7\% mais chance de ficarem retidos do que os discentes dos cursos diurnos.

\subsubsection{Desempenho acadêmico}

Para avaliar a influência do Coeficiente de Rendimento Acumulado (CRA) foi definido que a categoria "CRA de 9,01 a 10,00" seria tomada como referência. Dessa forma, observou-se que as chances de retenção de alunos com "CRA entre 8,01 e 9,00" é 1,9 vezes a daqueles da categoria de referência. Com "CRA entre 7,01 e 8,00" as chances de retenção são 3,8 vezes maiores, entre “6,01 e 7,00" são 5,1 vezes e entre " 4,01 e 6,00" são 10,1 vezes. As demais categorias não foram significantes.

Em relação ao número de reprovações, o modelo logístico apontou que o risco de retenção de alunos com "uma reprovação" é quase 2,3 vezes o daqueles que "não reprovaram" (referência). Para indivíduos com "duas a cinco reprovações", o risco é de 4,8 vezes, enquanto para aqueles com "seis ou mais reprovações" o risco é de 41,7 vezes. 


\subsubsection{Importância dos grupos de variáveis e de cada variável do modelo logístico}

Após a descrição das variações nas chances de retenção de alunos em função de mudanças ocorridas nas variáveis explicativas, foi investigada a importância relativa de cada grupo de variáveis dentro do modelo logístico gerado, por meio de outras quatro regressões logísticas multivariadas ${ }^{4}$.

Para tanto, foram utilizadas na geração dos modelos logísticos parciais somente as variáveis significantes incluídas no modelo logístico geral. O grau de importância do grupo foi definido com base no valor do $\mathrm{R}^{2}$ de Nagelkerke, utilizado como indicador do poder explicativo do modelo gerado com cada grupo de variáveis.

O grupo de variáveis que mais contribuiu para o poder explicativo do modelo logístico geral foi o "Desempenho Acadêmico". Este grupo, isoladamente, pode explicar 46,2\% das variações da variável dependente. Em seguida, os grupos de "Ambiente Institucional" e "Características pessoais e fatores anteriores ao ingresso" foram capazes de explicar, respectivamente, $26,3 \%$ e $21,1 \%$ das variações entre os grupos de alunos formados e retidos. O menor poder explicativo (3,9\%) foi observado no modelo que continha somente variáveis relativas ao "Contexto familiar e condições financeiras".

Em seguida, o grau de importância de cada uma das variáveis explicativas (significantes) também foi avaliado. Como base para essa avaliação, foi utilizado o valor do $\mathrm{R}^{2}$ de Nagelkerke dos modelos logísticos univariados gerados com cada uma das 21 variáveis incluídas no modelo logístico geral.

O modelo logístico gerado com a variável "Coeficiente de Rendimento Acumulado" possui o maior poder explicativo, sendo capaz de explicar $40,0 \%$ das variações ocorridas na variável dependente. O segundo maior poder explicativo $(38,2 \%)$ foi obtido com o modelo que considera, isoladamente, o "número de reprovações" em disciplinas. Além desses, completando o grupo de quatro modelos logísticos univariados de maior poder explicativo, constam aqueles gerados com as variáveis "Relação candidato/vaga do vestibular" (16,4\%) e "Área do conhecimento" (13,9\%). Essas variáveis são também aquelas que explicam mais de $10 \%$ das variações observadas na variável dependente e, por isso, podem ser consideradas as que mais contribuem para o poder explicativo do modelo logístico geral.

O método Enter foi adotado nesta etapa para que todas as variáveis significantes do modelo logístico geral sejam incluídas nos modelos parciais (modelos gerados a partir dos grupos de variáveis indicados na Seção 2.1). 


\subsection{Discussão dos resultados}

No modelo proposto para o estudo, assim como nas teorias de Astin (1984), Bean e Metzner (1985), Cabrera et al. (1992), Pascarella (1980), Spady $(1970,1971)$ e Tinto $(1975,1993,1997)$ o desempenho acadêmico (ou Resultados Acadêmicos) foi considerado o principal fator associado à permanência prolongada discente (e permanência ou evasão) no ensino superior. Essa proposição foi confirmada neste estudo, tendo em vista que, tanto agrupadas como isoladamente, as variáveis que representam o desempenho acadêmico ("Coeficiente de Rendimento Acumulado" e "Número de reprovações") foram as que apresentaram maior poder explicativo e as que mais contribuíram para a explicação obtida do modelo logístico geral.

Sobre a influência dessas variáveis no processo de permanência prolongada, alunos com maiores níveis de "Coeficiente de Rendimento Acumulado" possuem menor risco de ficarem retidos e, por outro lado, as reprovações reduzem o Coeficiente de Rendimento Acumulado e aumentam a chance de retenção.

Os demais grupos de variáveis ("Características pessoais e fatores anteriores ao ingresso", "Contexto familiar e condições financeiras" e "Ambiente Institucional") também foram significantes e contribuíram para a qualidade do modelo logístico gerado. Esse fato confirma que todos os grupos de variáveis incluídos no modelo teórico proposto têm participação no processo de permanência prolongada.

Dentre os quatro grupos de variáveis, o de "Contexto familiar e condições financeiras" foi aquele que se mostrou menos importante (menor poder explicativo). No entanto, a baixa influência dos fatores externos à instituição observada nesta pesquisa pode ser decorrente do uso de dados obtidos do questionário socioeconômico preenchido pelo aluno quando da sua inscrição para o vestibular. Assim, em virtude da dinamicidade das variáveis ligadas ao contexto familiar (envolvendo responsabilidades, condições financeiras e trabalho), parte dos dados pode ter sofrido alterações. Além disso, não foi possível incluir no estudo uma variável representativa da aprovação da família e amigos, apontada em diversos dos modelos teóricos revisados como importante fator para satisfação e comprometimento do aluno com a instituição.

Bean (1980) e Tinto (1975) consideram em suas teorias que diversas características pessoais e experiências anteriores ao ingresso na universidade também influenciam a satisfação e o comprometimento do aluno com seus objetivos educacionais e com a instituição. Essa influência foi identificada neste estudo através de três variáveis contidas no modelo logístico gerado: 
"O que espera em $1^{\circ}$ lugar do curso superior" (objetivos educacionais), "Motivo predominante para escolha do curso" e "Situação em relação a curso superior" (Escolaridade anterior).

Além dessas, as variáveis "Relação candidato/vaga" (concorrência) e "Desempenho no vestibular" (nas provas da $2^{\mathrm{a}}$ etapa) também correspondem a experiências do aluno antes do ingresso. $\mathrm{O}$ fato dos alunos que ingressaram em cursos de maior concorrência estarem sujeitos a um menor risco de retenção, pode estar associado a fatores como a necessidade de maior tempo de dedicação aos estudos e o desenvolvimento da habilidade de estudar para ser aprovado no vestibular. Esses fatores foram destacados por Bean e Metzner (1985) em seu modelo conceitual, mas não puderam ser verificados neste estudo.

Ainda sobre o desempenho no vestibular, os resultados deste estudo concordam com o que foi observado por Rios, Santos e Nascimento (2001) que indicaram que um baixo desempenho nas provas discursivas do vestibular está relacionado a um baixo desempenho no ciclo básico do curso e, consequentemente, pode levar o aluno à permanência prolongada.

Para Cabrera et al. (1992), o estudante com apoio financeiro tem melhores condições de integração social e acadêmica e assim maior chance de sucesso. Todavia a variável "Assistência Estudantil", incluída no estudo para representar a influência da ajuda financeira no processo, não alcançou o nível de significância estabelecido para compor o modelo logístico final. Esse fato não deve ser considerado conclusivo, tendo em vista que grande parte dos estudantes da amostra ingressou na instituição antes do início das ações de assistência. Essa situação demanda a realização de um estudo específico para investigar a influência da assistência estudantil no processo de permanência prolongada.

A permanência prolongada do aluno em razão de trancamentos de curso também foi relatada nos estudos de Polydoro (2000) e de Campello e Lins (2008). A forte associação entre o trancamento de curso e a retenção foi apontada pelo modelo logístico geral e pelo fato de $96,5 \%$ dos alunos analisados nesta pesquisa que trancaram o curso não se formaram no prazo esperado.

A realização de estágios, pelo menos parcialmente, está associada a um menor risco de retenção, ao passo que alunos que estagiaram por períodos entre $12 \mathrm{e}$ 24 meses têm $28,6 \%$ menos chance de ficar retidos do que aqueles que não o fizeram. Essa constatação contraria os resultados dos estudos de Campello e Lins 
(2008), Lautert, Rolim e Loder (2011) e Noronha, Carvalho e Santos (2001), que apontam o estágio como uma das principais causas da retenção discente. Dessa forma, a realização de estágios pode ser considerada um fator preventivo da permanência prolongada.

A influência das reprovações e da realização de intercâmbio na retenção dos discentes observada neste estudo foi relatada anteriormente por Campello e Lins (2008) e Corrêa e Noronha (2004). Nesses dois estudos, tanto as reprovações, como a realização de intercâmbio, favoreceram a ocorrência de retenção.

\subsection{Propostas de intervenção institucional}

O foco das ações institucionais deve ser a melhoria do desempenho acadêmico dos estudantes, sem perder de vista que este é resultado de um conjunto de fatores internos e externos à instituição, além do comportamento do discente, conforme apontando nas teorias da área.

Considerando os principais fatores relevantes no processo de permanência prolongada, recomenda-se a realização de novas pesquisas sobre desempenho acadêmico, reprovações e trancamentos de curso buscando conhecer as causas, os fatores envolvidos e as interpretações de alunos e docentes. Os resultados dessas pesquisas devem ser utilizados para subsidiar a elaboração de novos projetos de pesquisa e de ações institucionais.

Uma segunda proposta é a estruturação de programas de suporte acadêmico ao estudante, tais como tutoria e orientação acadêmica, bem a reestruturação do programa de monitoria com objetivo de contribuir para superação de dificuldades pré-existentes, apoiar o aprendizado e a obtenção de bom desempenho. Proposições como essa foram apresentadas nos estudos de Rios, Santos e Nascimento (2001), Soares (2006) e Campello e Lins (2008).

O efeito preventivo da realização de estágio sobre o risco de retenção discente indica que o incentivo a programas de estágio e divulgação de ofertas de vagas também são ações institucionais aplicáveis para redução dos níveis de permanência prolongada.

A aproximação da universidade com a sociedade, especialmente as escolas de ensino médio, almejando a atração de futuros alunos com perfil adequado a instituição e seus cursos, é uma possível intervenção defendida por Noronha, Carvalho e Santos (2001). 
A adoção do indicador "Nível de retenção baseado no ritmo do aluno", proposto por Dias, Cerqueira e Lins (2009), constitui mais uma proposta de ação institucional. A informação sobre o nível de retenção do aluno pode ser disponibilizada aos coordenadores de curso e professores, permitindo assim a identificação e acompanhamento de casos de retenção excessiva e possibilitando a adoção de medidas preventivas e corretivas a cada semestre.

\section{Considerações finais}

O objetivo desta pesquisa foi identificar os fatores associados à permanência prolongada dos estudantes dos cursos de graduação presenciais de universidade federal brasileira e propor ações para combater o problema.

Com esse foco foram identificados 21 fatores que influenciam o processo de permanência prolongada, dos quais seis são relativos a "Características pessoais e fatores anteriores ao ingresso" na universidade, quatro são do grupo "Contexto familiar e condições financeiras", nove são do "Ambiente Institucional" e duas representam "Desempenho Acadêmico" do estudante. Esse resultado revelou a complexidade do processo estudado.

Além disso, foi constatado que o grupo de variáveis mais importante foi o "Desempenho Acadêmico", seguido dos grupos "Ambiente Institucional" e "Características pessoais e fatores anteriores ao ingresso" e "Contexto familiar e condições financeiras", nesta ordem. Já os fatores mais importantes foram "Coeficiente de Rendimento Acumulado", "Número de reprovações", "Relação candidato/vaga", "Área do Conhecimento", "Desempenho no vestibular" e "Trancamento de curso".

A partir dos resultados obtidos foram apresentadas algumas propostas de intervenção institucional visando à redução dos níveis de retenção na universidade. Uma delas é a realização de novas pesquisas sobre desempenho acadêmico, reprovações e trancamentos de curso, buscando compreender as razões dos problemas de desempenho, do elevado número de reprovações e dos trancamentos de curso.

Foi proposta também a estruturação de programas de suporte acadêmico ao estudante, tais como tutoria, monitoria e orientação acadêmica, bem como o incentivo a realização de estágios. Outras propostas foram a de aproximação da universidade com a sociedade, especialmente as escolas de ensino médio e a utilização de um indicador de monitoramento do nível de retenção dos alunos. 
Uma das limitações do estudo refere-se ao uso de dados obtidos do questionário socioeconômico aplicado no vestibular para representar o contexto familiar e condições financeiras. Parte dos dados utilizados pode ter sofrido alterações, tendo em vista a dinâmica dessas condições (responsabilidades, condições financeiras e de trabalho). Somado a isso, não foi possível incluir no estudo uma variável representativa da aprovação da família e amigos, apontada em vários modelos teóricos como importante fator para satisfação e comprometimento do aluno com a instituição.

Mesmo com as limitações impostas e a complexidade do fenômeno de retenção discente nos cursos superiores, este estudo contribuiu para o aumento da compreensão do processo, ao passo que identificou fatores intervenientes e suas influências sobre o risco de permanência prolongada. Destaca-se que, nesta pesquisa, a retenção discente ou permanência prolongada foi o objeto central, diferentemente do que se observa na maioria dos estudos da área que privilegiam a evasão.

Ademais, o procedimento metodológico adotado na pesquisa pode ser empregado em outras instituições de ensino superior; contudo, os resultados poderão variar, porquanto algumas variáveis podem ter comportamento distinto do que se observou na UFES.

Os resultados obtidos permitiram ainda observar as similaridades que existem entre os processos de permanência prolongada e evasão. Por isso, acredita-se que os modelos teóricos concebidos para estudo da evasão sejam também aplicáveis à retenção e que as ações institucionais propostas têm potencial de reduzir tanto os níveis de permanência prolongada como os de abandonos.

\section{Agradecimentos}

Os autores agradecem a contribuições de Jaime Sales Souza Junior, Giany do Nascimento Terra e João Luiz Calmon. 


\section{The effects of prolonged higher education: a study on the Federal University of Espírito Santo}

\section{Abstract}

Prolonged higher education is the condition in which a student requires longer time to complete all the coursework for graduation. At the Federal University of Espirito Santo, $52.2 \%$ of the students who should have graduated between February 2007 and January 2012 were retained. Therefore, the purpose of this study is to identify the factors associated with the retention of students and propose institutional actions to resolve this problem. The methodology consists of comparing two groups of students (retained and graduated) by means of a logistic regression model. The results indicate that 21 factors were involved in the process, of which the most significant included the following: the accumulated coefficient of performance, the number of failures; the ratio of applicants/vacancies in the entrance examination, the knowledge of the subjects, the performance in the second stage of the entrance examination, and the process of completing the coursework.

Keywords: Higher education. Student retention. Prolonged higher education. Logistic regression.

\section{Factores importantes en el proceso de permanencia prolongada de estudiantes en los cursos de educación superior presencial: un estudio en la Universidad Federal de Espírito Santo}

\section{Resumen}

Permanencia prolongada de los estudiantes en el sistema de educación superior es la condición en la que el estudiante requiere más tiempo de lo esperado en el plan de estudios para concluir el curso. En la Universidad Federal de Espírito Santo, el $52,2 \%$ de los estudiantes que deberían concluir los cursos entre los semestres 2007/2 a 2012/1 se retrasaron en el sistema. El objetivo de este estudio fue identificar los factores asociados con el retraso en los cursos de educación superior presenciales, de manera de proponer acciones institucionales para combatir el problema. La metodología consistió en la comparación de dos grupos de estudiantes, los retrasados y los que concluyeron el curso de acuerdo con el plan de estudios, a través de un modelo de regresión logística. Los resultados indicaron 21 factores que intervienen en el proceso, de los cuales, los más relevantes fueron: el indicador de desempeño acumulado del estudiante, número de asignaturas suspendidas, relación candidato por plaza en la selección, el área de conocimiento científico, el desempeño en los exámenes de la segunda etapa en la selección y la solicitud de excedencia por periodo definido en normativas de la universidad.

Palabras clave: Educación superior presencial. Retraso de los estudiantes. Permanencia prolongada. Regresión logística. 


\section{Referências}

ASTIN, A. W. Student involvement: a developmental theory for higher education. Journal of College Student Personnel, Washington, v. 25, p. 297-308, 1984.

BEAN, J. P. Dropout and turnover: the synthesis and test of a causal model of student attrition. Research in Higher Education, New York, v. 12, p. 155-87. 1980.

BEAN, J. P.; METZNER, B. S. A conceptual model of nontraditional undergraduate student attrition. Review of Educational Research, Washington, v. 55 , p. $485-540,1985$.

BRASIL. Ministério da Educação. Secretaria da Educação Superior - SESu. Diplomação, retenção e evasão nos cursos de graduação em Instituições de Ensino Superior Públicas: relatório da Comissão Especial de Estudos sobre Evasão nas Universidades Públicas Brasileiras. Brasília, DF: MEC, 1997.

CABRERA, A. F. et al. The convergence between two theories of college persistence. Journal of Higher Education, Columbus, v. 63, n. 2, p. 143-64, 1992.

CAMPELLO, A. V. C.; LINS, L. N. Metodologia de análise e tratamento da evasão e retenção em cursos de graduação de instituições federais de ensino superior. In: ENCONTRO NACIONAL DE ENGENHARIA DE PRODUCÃO, 28., 2008, Rio de Janeiro. Anais eletrônicos...

Disponível em: $<$ http://www.abepro.org.br/biblioteca/ enegep2008_TN_ STO_078_545_11614.pdf $>$. Acesso em: 21 mar. 2013.

CORRAR, L.J.; PAULO, E.; DIAS FILHO, J.M. (Coord.) Análise multivariada: para os cursos de administração, ciências contábeis e economia. São Paulo: Atlas, 2007.

CORRÊA, A. C. C.; NORONHA, A. B. Avaliação da evasão e permanência prolongada em um curso de graduação em administração de uma universidade pública. In: SEMINÁRIOS EM ADMINISTRAÇÃO FEA-USP, 7., 2004, São Paulo. Anais eletrônicos... Disponível em: <http://www.ead.fea.usp.br/ Semead/7Semead/paginas/artigos recebidos/Ensino/ENS10__Avaliacao_da_ evasao_e_permanencia_prol.PDF>. Acesso em: 10 mar. 2013.

DIAS, A. F. M.; CERQUEIRA, G. S.; LINS, L. N. Fatores determinantes da retenção estudantil em um curso de graduação em engenharia de produção. In: CONGRESSO BRASILEIRO DE EDUCAÇÃO EM ENGENHARIA, 37. 2009, Recife. Anais eletrônicos... Disponível em: <http://www.abenge.org.br/ CobengeAnteriores/2009/artigos/682.doc>. Acesso em: 14 mar. 2013. 
FÁVERO, L. P. et al. Análise de dados: modelagem multivariada para tomada de decisões. Rio de Janeiro: Elsevier, 2009.

FIELD, A. Descobrindo a estatística usando o SPSS. 2. ed. Porto Alegre: Artmed. 2009.

HAIR, F. J. et al. Análise multivariada de dados. 6. ed. Porto Alegre: Bookman, 2009.

LAUTERT, L. V. S.; ROLIM, M.; LODER, L. L. Investigando processos de retenção no âmbito de um curso de engenharia elétrica. In: CONGRESSO BRASILEIRO DE EDUCAÇÃO EM ENGENHARIA, 39., 2011, Blumenau. Anais eletrônicos... Disponível em: <http:// www.abenge.org.br/CobengeAnteriores/2011/sessoestec/art2094.pdf>. Acesso em: 20 mar. 2013.

NORONHA, A. B.; CARVALHO, B. M.; SANTOS, F. F. F. Estudo do perfil dos alunos evadidos da faculdade de economia, administração e contabilidade, campus Ribeirão Preto, e avaliação do tempo de titulação dos alunos atualmente matriculados. Ribeirão Preto: FEA/USP, 2001. (Texto para Discussão. Série Administração).

PASCARELLA, E. T. Student-faculty informal contact and college outcomes. Review of Educational Research, Washington, v. 50, n. 4, p. 545-95, 1980.

PEREIRA, A. S. Retenção discente nos cursos de graduação presencial da UFES. 2013. 164 f. Dissertação (Mestrado Profissional em Gestão Pública)-Programa de Pós-Graduação em Gestão Pública, Universidade Federal do Espírito Santo, Vitória, 2013.

POLYDORO, S. A. J. O trancamento de matrícula na trajetória acadêmica do universitário: condições de saída e de retorno à instituição. $2000.179 \mathrm{f}$. Tese (Doutorado em Educação)-Programa de Pós-Graduação em Educação, Universidade Estadual de Campinas, Campinas, 2000.

RIOS, J. R. T.; SANTOS, A. P.; NASCIMENTO, C. Evasão e retenção no ciclo básico dos cursos de engenharia da Escola de Minas da UFOP. In: CONGRESSO BRASILEIRO DE ENSINO DE ENGENHARIA, 29., 2001, Porto Alegre. Anais eletrônicos... Disponível em: <http:// www.abenge.org.br/CobengeAnteriores/2001/trabalhos/APP020.pdf>. Acesso em: 21 mar. 2013. 
SOARES, I. S. Evasão, retenção e orientação acadêmica: UFRJ - Engenharia de Produção: estudo de caso. In: CONGRESSO BRASILEIRO DE EDUCAÇÃO EM ENGENHARIA, 34., 2006, Passo Fundo. Anais eletrônicos... Disponível em: $<\mathrm{http}: / / \mathrm{www}$.abenge.org.br/ CobengeAnteriores/2006/artigos/8_228_956.pdf >. Acesso em: 2 fev. 2013.

SPADY, W. G. Dropouts from higher education: an interdisciplinary review and synthesis. Interchange, Toronto, v. 1, p. 64-85, 1970.

. Dropouts from higher education: toward an empirical model. Interchange, Toronto, v. 2, n. 3, p. 38-62. 1971.

TINTO, V. Dropout from higher education: a theoretical synthesis of recent research. Review of Educational Research, Washington, v. 45, n. 1, p. 89-125, 1975.

. Classrooms as communities: exploring the educational character of student persistence. Journal of Higher Education, Columbus, v. 68, n. 6, p. 599-624, 1997.

. Leaving college: rethinking the causes and cures of student attrition. 2. ed. Chicago: University of Chicago Press, 1993.

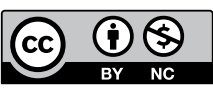

\section{Informações dos autores}

Alexandre Severino Pereira: Mestrado em Gestão Pública. Diretor da Divisão de Logística e Infraestrutura da Pró-Reitoria de Gestão de Pessoas e Assistência Estudantil, Universidade Federal do Espírito Santo - UFES. Contato: alexandre.severino.@gmail.com

Teresa Cristina Janes Carneiro: Doutorado em Administração, Instituto de Pós-Graduação e Pesquisa em Administração/Universidade Federal do Rio de Janeiro - COPPEAD/UFRJ. Coordenadora do Mestrado Profissional em Gestão Pública, Universidade Federal do Espírito Santo - UFES. Coordenadora da Universidade Aberta do Brasil/Universidade Federal do Espírito Santo - UAB/UFES. Membro do Comitê Gestor e Coordenadora da Comissão Acadêmica Nacional - PROFIAP. Mestrado Profissional em Administração Pública em rede nacional. Contato: carneiro.teresa@gmail.com

Gutemberg Hespanha Brasil: Doutorado em Engenharia Elétrica, Pontifícia Universidade Católica do Rio de Janeiro - PUC-Rio. Professor associado III , Universidade Federal do Espírito Santo - UFES. Contato: ghbrasil@terra.com.br

Maria Auxiliadora de Carvalho Corassa: Doutorado em Comunicação e Semiótica, Pontifícia Universidade Católica de São Paulo - PUC-SP. Professor adjunto IV e Pró-reitora de Graduação, Universidade Federal do Espírito Santo - UFES'. Contato: dora.ddp@gmail.com 